\title{
Compound haploinsufficiency of Dok2 and Dusp4 promotes lung tumorigenesis
}

\author{
Ming Chen, ${ }^{1}$ Jiangwen Zhang, ${ }^{2}$ Alice H. Berger, ${ }^{1}$ Moussa S. Diolombi, ${ }^{1}$ Christopher $\mathrm{Ng},{ }^{1}$ Jacqueline Fung, ${ }^{1}$ Roderick T. Bronson, ${ }^{3}$ \\ Mireia Castillo-Martin, ${ }^{4,5}$ Tin Htwe Thin, ${ }^{4}$ Carlos Cordon-Cardo, ${ }^{4}$ Robin Plevin, ${ }^{6}$ and Pier Paolo Pandolfi ${ }^{1}$ \\ 'Cancer Research Institute, Beth Israel Deaconess Cancer Center, Department of Medicine and Pathology, Beth Israel Deaconess Medical Center (BIDMC), Harvard Medical School, Boston, Massachusetts, \\ USA. ${ }^{2}$ School of Biological Sciences, The University of Hong Kong, Hong Kong, China. ${ }^{3}$ Department of Microbiology and Immunobiology, Harvard Medical School, Boston, Massachusetts, USA. ${ }^{4}$ Department of \\ Pathology, Icahn School of Medicine at Mount Sinai, New York, New York, USA. ${ }^{5}$ Department of Pathology, Champalimaud Center for the Unknown, Lisbon, Portugal. ${ }^{6}$ Strathclyde Institute of Pharmacy and \\ Biomedical Sciences, University of Strathclyde, Glasgow, United Kingdom.
}

Recurrent broad-scale heterozygous deletions are frequently observed in human cancer. Here we tested the hypothesis that compound haploinsufficiency of neighboring genes at chromosome 8p promotes tumorigenesis. By targeting the mouse orthologs of human DOK2 and DUSP4 genes, which were co-deleted in approximately half of human lung adenocarcinomas, we found that compound-heterozygous deletion of Dok2 and Dusp4 in mice resulted in lung tumorigenesis with short latency and high incidence, and that their co-deletion synergistically activated MAPK signaling and promoted cell proliferation. Conversely, restoration of DOK2 and DUSP4 in lung cancer cells suppressed MAPK activation and cell proliferation. Importantly, in contrast to downregulation of DOK2 or DUSP4 alone, concomitant downregulation of DOK2 and DUSP4 was associated with poor survival in human lung adenocarcinoma. Therefore, our findings lend in vivo experimental support to the notion that compound haploinsufficiency, due to broad-scale chromosome deletions, constitutes a driving force in tumorigenesis.

\section{Introduction}

Human cancers are characterized by recurrent somatic copy number alterations (SCNAs), which include both focal and broad arm-level amplifications and deletions (1). How these alterations contribute to tumorigenesis remains a central question in cancer research. Specifically, large heterozygous deletions are thought to produce a "second hit" toward the inactivation of a tumor suppressor gene (TSG), hence fulfilling Knudson's two-hit hypothesis (2). However, TSGs that fulfill this paradigm are often not identified in chromosomal loci displaying consistent loss of heterozygosity $(\mathrm{LOH})(1,3)$, calling into question whether bona fide TSGs actually reside in those regions. One possible explanation is that these deletions arise from genomic instability and are inconsequential to tumor formation, but another plausible explanation is that haploinsufficiency of 2 or more TSGs in the deleted regions cooperates to promote tumorigenesis (4-9).

Large deletions of chromosome $8 \mathrm{p}$ are common in a variety of tumor types, including breast, colon, liver, and lung cancer and leukemia (9-12). A region of $8 \mathrm{p}$ loss, spanning cytobands $8 \mathrm{p} 12$ $8 \mathrm{p} 23$, is frequently observed in human lung adenocarcinoma (11, 13), and multiple putative TSGs have been proposed in this region $(9,13,14)$. Using a knockout mouse model, we previously identified the $8 \mathrm{p} 21$ gene DOK2, encoding an adaptor protein that mod-

Conflict of interest: The authors have declared that no conflict of interest exists. License: Copyright 2019, American Society for Clinical Investigation.

Submitted: January 8, 2018; Accepted: October 9, 2018.

Reference information: J Clin Invest. 2019;129(1):215-222.

https://doi.org/10.1172/JCI99699. ulates the RAS/RTK signaling pathway, as a human lung tumor suppressor (14). Human genetics and in vitro functional data have also identified DUSP4, encoding a dual-specificity phosphatase that functions as part of a negative feedback loop to terminate the activity of MAPKs, as another candidate lung TSG on $8 p$ (11). Notably, deletions of $8 p$ in human lung adenocarcinoma frequently encompass both DOK2 and DUSP4, and are enriched in tumors with EGFR mutations $(11,15)$.

Given the genomic localization of DOK2 and DUSP4 and their convergent function in suppression of MAPK signaling, we hypothesized that compound haploinsufficiency of DOK2 and DUSP4 promotes lung tumorigenesis. Here we show that although heterozygous or complete loss of Dusp4 is not sufficient to initiate lung tumorigenesis in mice, heterozygous loss of Dusp 4 markedly accelerates lung tumorigenesis in Dok2heterozygous mice. In agreement with these findings, we find that concomitant downregulation of DOK2 and DUSP4 is associated with poor survival in human lung adenocarcinoma.

\section{Results}

Dusp4 and Dok2 heterozygosity cooperate to promote lung tumorigenesis in vivo. Human genetics and in vitro functional studies previously identified DUSP4 as a putative 8p12 TSG in human lung adenocarcinoma (11). To study the impact of Dusp4 loss on lung tumorigenesis in mice, we analyzed Dusp4-KO mice generated by a targeted deletion strategy (16). Unlike Dok2 mutant mice (14), Dusp $4^{+/-}$and Dusp4 $4^{-/-}$mice did not develop lung cancer within a 12- or 18-month follow-up (Figure 1, A and B, and Table 1), indicating that loss of Dusp4 alone in mice is not sufficient to initiate lung tumorigenesis in vivo. 

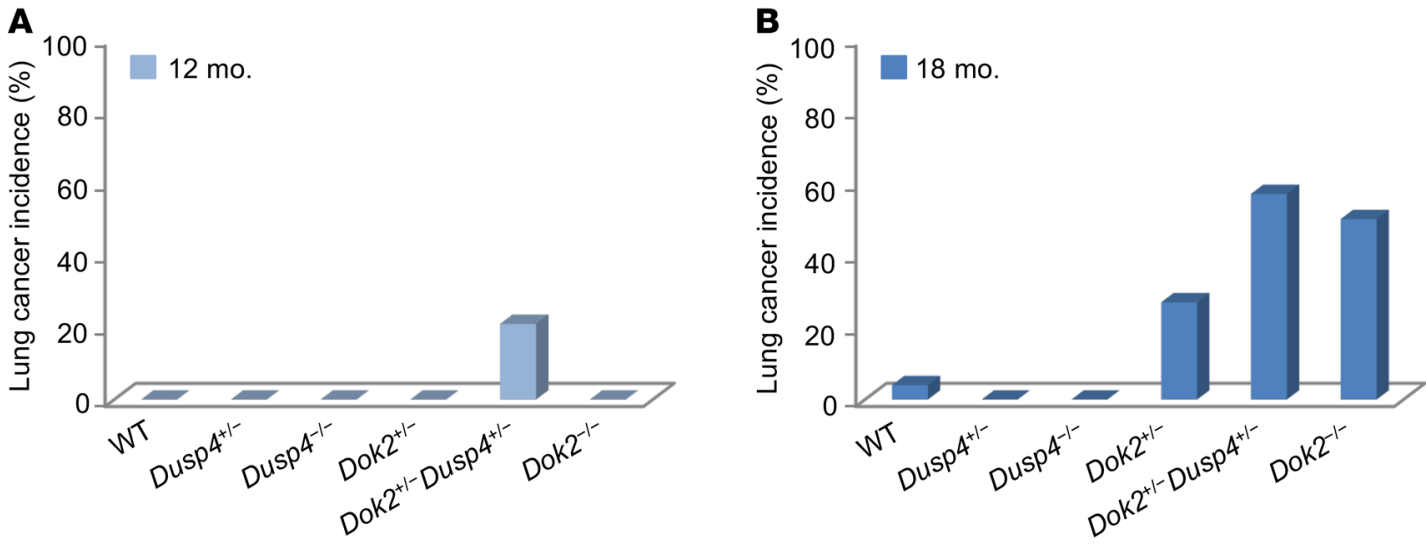

Figure 1. Compound heterozygous loss of Dok2 and Dusp4 in mice induces lung cancer. (A and B) Lung adenocarcinoma incidence in Dusp4 and Dok2 heterozygous-, homozygous-, and compound heterozygous-KO mice at 12 months of age (A) and at 18 months of age (B). Mouse numbers and statistics are summarized in Table 1.

We previously identified DOK2 as a candidate human lung TSG (14). $30 \%$ of Dok2 heterozygous (Dok2+/-) mice developlung adenocarcinoma, albeit with a long latency of 15-19 months. Tumors from these mice do not lose the WT Dok2 allele, suggesting that Dok2 is a haploinsufficient TSG. Thus, we sought to determine whether haploinsufficiency of Dusp4 impacts lung tumorigenesis initiated by Dok2 haploinsufficiency in vivo. We crossed the Dusp $4^{+/-}$mice with Dok2 ${ }^{+/-}$mice to generate mice compound-heterozygous for Dok2 and Dusp4 (Dok2 $2^{+/-}$Dusp $4^{+/-}$ mice) and compared the incidence of lung adenocarcinoma in single- and compound-mutant mice at 12 and 18 months of age. At 12 months, Dok2 ${ }^{+/-}$Dusp $4^{+/-}$mice were the only genotype of mice that developed lung adenocarcinoma at moderate $(21 \%)$ penetrance (Figure 1A and Table 1). Further examination of lungs of the younger Dok2 $2^{+/-} \mathrm{Dusp} 4^{+/-}$mice revealed that as early as 9 months of age, $20 \%$ of Dok2 ${ }^{+/-}$Dusp $4^{+/-}$mice developed lung tumors (Supplemental Figure 1A, $n=10$ ). At 18 months, an analysis of 157 mice showed that Dok2 ${ }^{+/-}$Dusp $4^{+/-}$mice had a significantly higher incidence of lung adenocarcinoma than Dok2 $2^{+-}$mice (Figure 1B and Table 1).

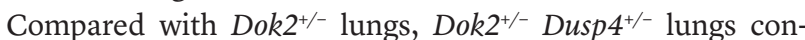
tained significantly larger tumor nodules (Figure 2A). Tumors in
Dok2 $2^{+/-}$Dusp $4^{+/-}$mice were adenocarcinomas with solid and papillary growth patterns (Supplemental Figure 1B), similar to lung tumors in Dok-mutant mice (14). However, they had a significantly elevated frequency of mitotic cells positive for Ki-67 staining when compared with $D o k 2^{+-}$lung tumors (Figure 2B). Given the role of DOK2 and DUSP4 in the regulation of RTK signaling, we conducted IHC to determine the levels of phosphorylated MAPK/ Erk in the murine tumors. Dok2+/- lung tumors showed moderate staining for phosphorylated Erk (Figure 2C), while Dok2 ${ }^{+/-} \mathrm{Dusp} 4^{+/-}$ lung tumors showed stronger staining for phosphorylated Erk than Dok2 $2^{+-}$lung tumors (Figure 2C), which was confirmed by Western blot analysis (Figure 2D). In contrast, lung tissues from WT and Dusp4-mutant mice showed low or undetectable staining for Ki-67 and phosphorylated Erk (Supplemental Figure 1C). Thus, in contrast to heterozygous loss of Dok2, heterozygous loss of Dusp4 did not initiate lung tumorigenesis, but instead accelerated the progression of tumors initiated by Dok2 heterozygosity, possibly through augmentation of MAPK activation.

Importantly, IHC staining showed that protein expression of both Dok2 and Dusp4 was retained in the Dok2 $2^{+-}$Dusp $4^{+/-}$ lung tumors, indicating that complete genetic loss of either Dok2 or Dusp4 is not required for tumor development (Figure 2E).

Table 1. Incidence of lung adenocarcinoma in cohorts of WT, Dok2-, Dusp4-, and compound-mutant mice

\begin{tabular}{|c|c|c|c|c|c|c|c|c|}
\hline \multirow[b]{3}{*}{ Genotype } & \multicolumn{8}{|c|}{ Age of mice } \\
\hline & \multicolumn{4}{|c|}{12 months } & \multicolumn{4}{|c|}{18 months } \\
\hline & $\boldsymbol{n}_{\text {cancer }}$ & $n_{\text {total }}$ & $\%$ with cancer & 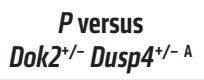 & $\boldsymbol{n}_{\text {cancer }}$ & $n_{\text {total }}$ & $\%$ with cancer & $\begin{array}{c}\text { P versus Dok2 } 2^{+/-} \\
\text {Dusp4 }^{+/-\mathrm{A}}\end{array}$ \\
\hline WT & 0 & 21 & 0 & 0.031 & 1 & 25 & $4 \%$ & $<0.001$ \\
\hline Dusp4 $4^{+/-}$ & 0 & 22 & 0 & 0.028 & 0 & 24 & 0 & $<0.001$ \\
\hline Dusp4-/- & 0 & 29 & 0 & 0.010 & 0 & 24 & 0 & $<0.001$ \\
\hline Dok2+/- & 0 & 23 & 0 & 0.027 & 7 & 26 & $27 \%$ & 0.032 \\
\hline Dok2 $2^{+/-}$Dusp4 $4^{+/-}$ & 6 & 28 & $21 \%$ & - & 17 & 30 & $57 \%$ & - \\
\hline Dok2-/- & 0 & 24 & 0 & 0.025 & 14 & 28 & $50 \%$ & 0.793 \\
\hline
\end{tabular}

AThe incidence of lung adenocarcinoma in Dok2 and Dusp4 mutant mice at 12 and 18 months of age, respectively, was compared with that of compound Dok2 and Dusp4 heterozygous mice using Fisher's exact test; $P<0.05$ was considered significant. 
A

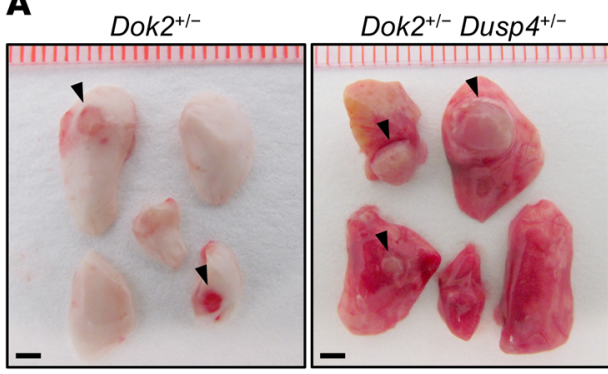

B

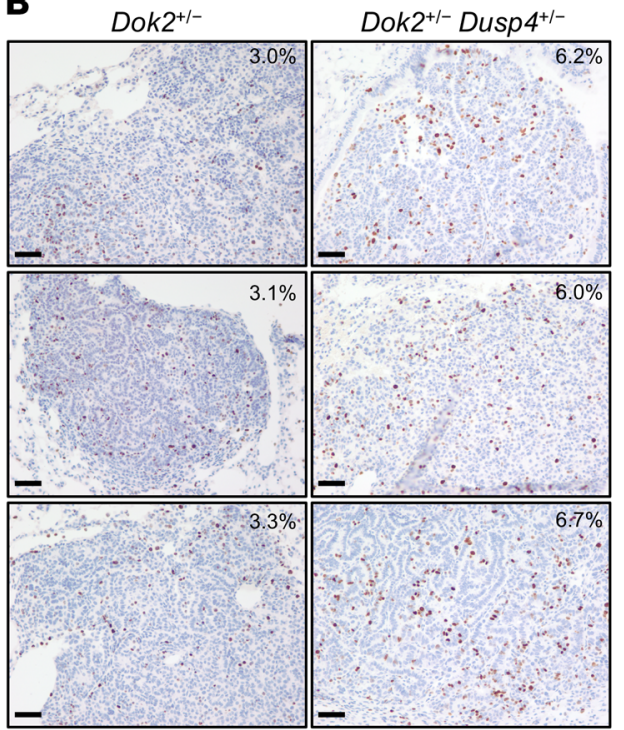

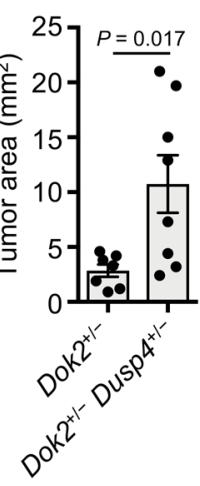

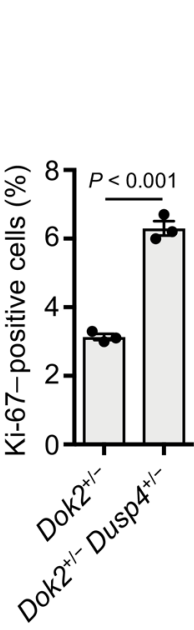

C

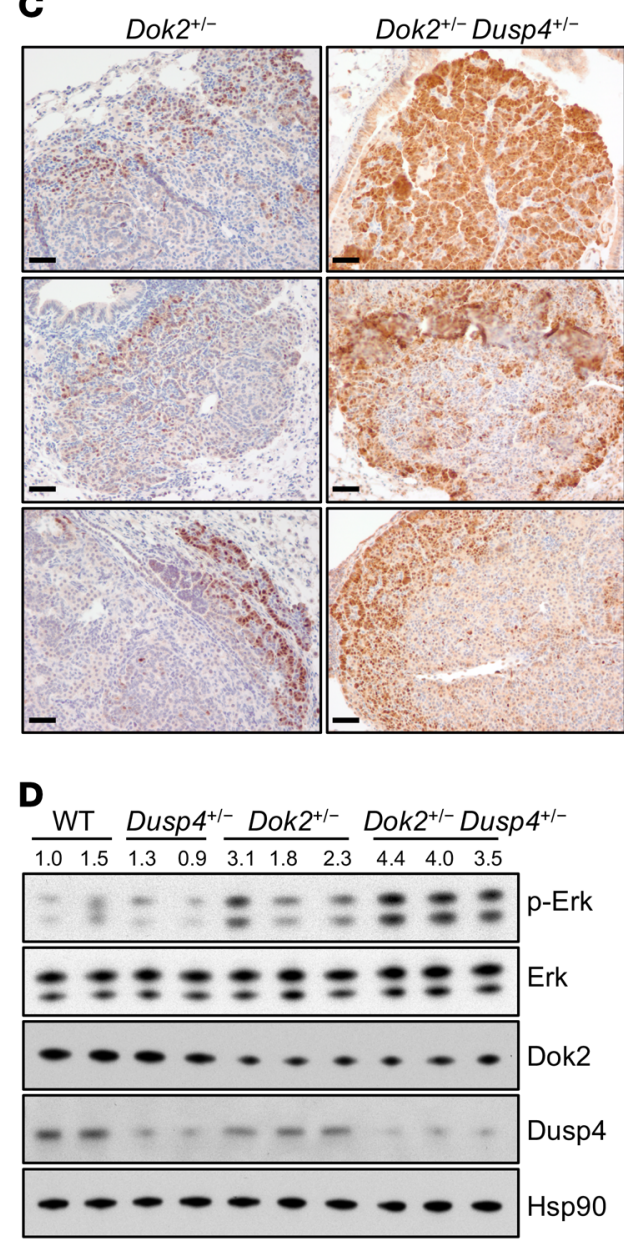

E

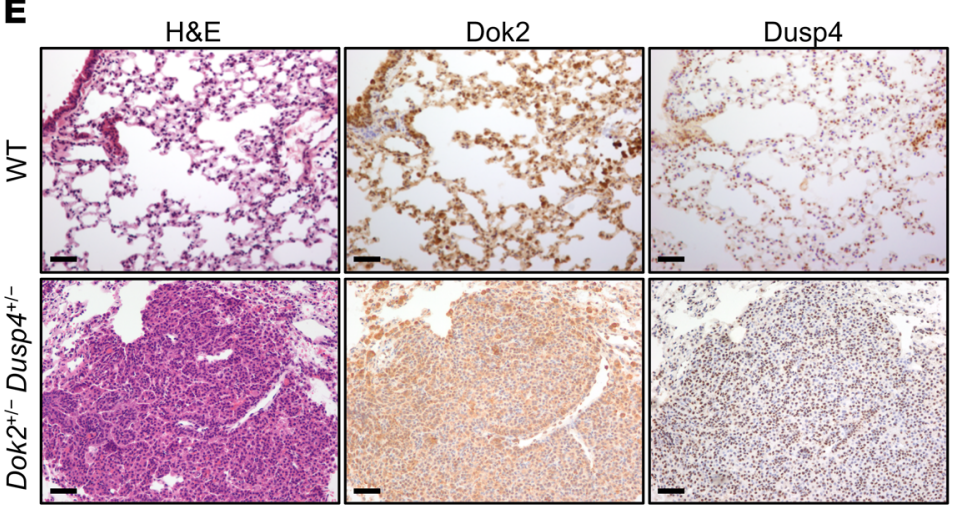

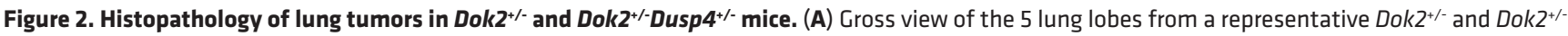
Dusp4 $4^{+-}$mouse. Arrowheads indicate tumor nodules. Quantification of the size of tumors was carried out with Image) software ( $n=5$ mice per genotype).

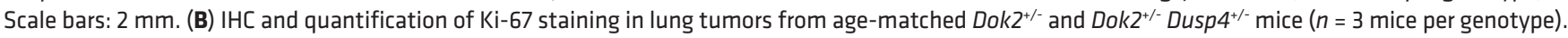

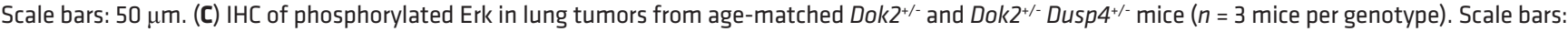
$50 \mu \mathrm{m}$. (D) Western blot analysis of lung lysates from 9-month-old WT, Dusp4 ${ }^{+/}$, Dok2 $2^{+/}$, and Dok2 $2^{+-}$Dusp4 ${ }^{+/-}$mice. Numbers indicate the ratios relative to controls for phospho-protein/total protein. (E) Serial sections of lung tissue stained with H\&E and IHC for Dok2 and Dusp4 from an 18-month-old WT and Dok2 ${ }^{+-}$Dusp4 $4^{+-}$mouse. Scale bars: $50 \mu \mathrm{m}$. In A and B, data are shown as mean \pm SEM. Student's $t$ test (2 tailed) was used to determine significance.

Therefore, Dok2 and Dusp4 are haploinsufficient in their tumorsuppressive function and their ability to suppress MAPK activation.

DOK2 and DUSP4 cooperate to suppress MAPK activation and cell proliferation. To assess the tumor-suppressive role of DOK2 and DUSP4 in human lung cancer, we stably overexpressed DOK2 or/and DUSP4 in 3 lung cancer cell lines, H1650, H1975, and H3255, all characterized by EGFR mutation and loss of one copy of DOK2 and DUSP4 (Supplemental Table 1; data from Cancer Cell Line Encyclopedia hosted on cBioPortal) (17). Overexpression of either DOK2 or DUSP4 significantly suppressed MAPK activation, 

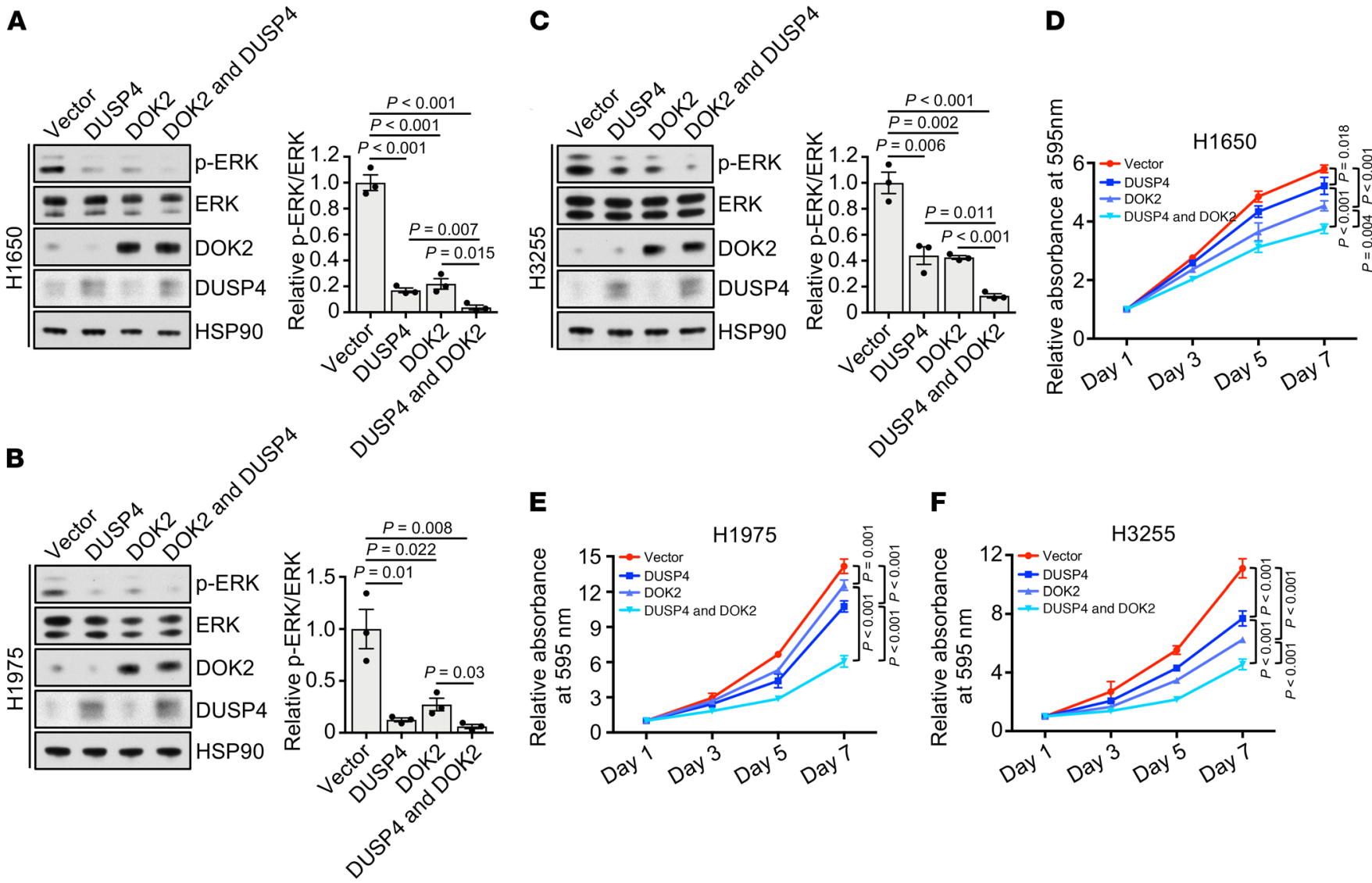

$\mathbf{E}$

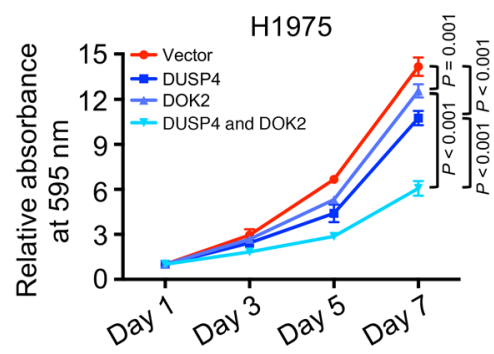

D

$\mathbf{F}$

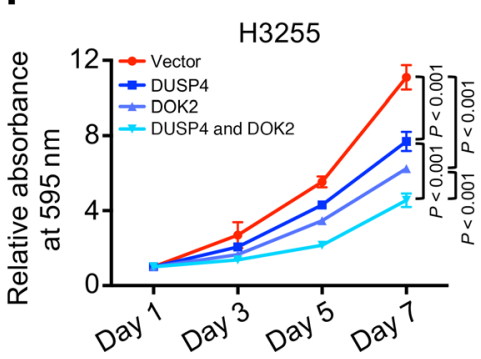

G
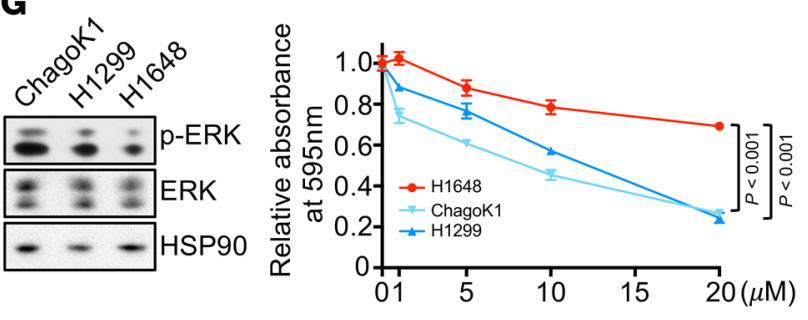

Figure 3. DOK2 and DUSP4 cooperate to suppress lung cancer cell proliferation in vitro. (A-C) Western blot of cell lysates from lung cancer cell lines H1650 (A), H1975 (B), and H3255 (C) stably expressing vector, DOK2, or/and DUSP4. Quantification of p-ERK/ERK levels was carried out with ImageJ software $(n=3$ experiments). Numbers indicate the ratios relative to controls for phosphoprotein/total protein. Data are shown as mean \pm SEM. Student's $t$ test (2 tailed) was used to determine significance. (D-F) Growth curve analysis of lung cancer cell lines H1650 (D), H1975 (E), and H3255 (F) stably expressing vector, DOK2, or/and DUSP4. Relative cell number was determined after cell fixation and crystal violet staining using OD at $595 \mathrm{~nm}$. Data were normalized against the OD value on day 1 of each cell line. Data are shown as mean \pm SD of a representative experiment $(n=3$ experiments). Two-way ANOVA with Bonferroni's post hoc test was used to determine significance. (C) Growth curve analysis of lung cancer cell lines ChagoK1, H1299, and H1648 treated with various doses of the MEK inhibitor U0126 for 7 days. Relative cell number was determined after cell fixation and crystal violet staining using OD at $595 \mathrm{~nm}$. Western blot analysis indicated the expression of $\mathrm{p}$-ERK. Data were normalized against the OD value of vehicle-treated cells. Data are shown as mean \pm SEM of a representative experiment ( $n=3$ experiments). Two-way ANOVA with Bonferroni's post hoc tests were used to determine significance.

and combined expression of DOK2 and DUSP4 suppressed MAPK to the greatest degree in H1650 and H3255 cell lines (Figure 3, $\mathrm{A}-\mathrm{C}$ ). Consistent with these findings, in a growth curve assay, cells expressing either DOK2 or DUSP4 displayed an impaired growth rate compared with cells transfected with empty vector (Figure 3, D-F). Combined expression of DOK2 and DUSP4 further impaired cell growth compared with either DOK2 or DUSP4 overexpression (Figure 3, D-F). These data further support a cooperative role of DOK2 and DUSP4 in lung tumor suppression.

Compound heterozygosity of DOK2 and DUSP4 confers enhanced sensitivity to MEK inhibition. The observation that DOK2 and DUSP4 converge on the suppression of MAPK signaling led us to investigate whether cells with one copy loss of DOK2 and DUSP4 might exhibit enhanced sensitivity to MEK inhibition compared with cells with intact copies of DOK2 and DUSP4. To avoid confounding effects of MAPK activity induced by mutant EGFR or KRAS, we examined the response to MEK inhibition in cells with WT EGFR and KRAS with either one copy loss of DOK2 and DUSP4 (ChagoK1 and H1299) or no loss of DOK2 and DUSP4 (H1648) (Supplemental Table 1). We found that both ChagoK1 and H1299 cells exhibited higher ERK activation and were more sensitive to MEK inhibition than H1648 cells (Figure 3G). These 
A

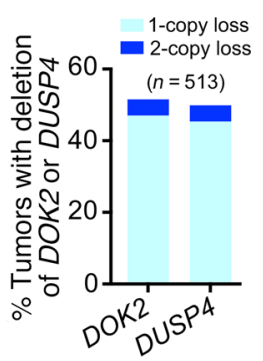

B

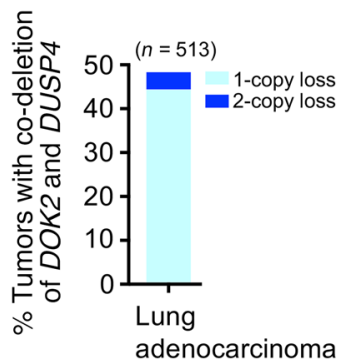

C

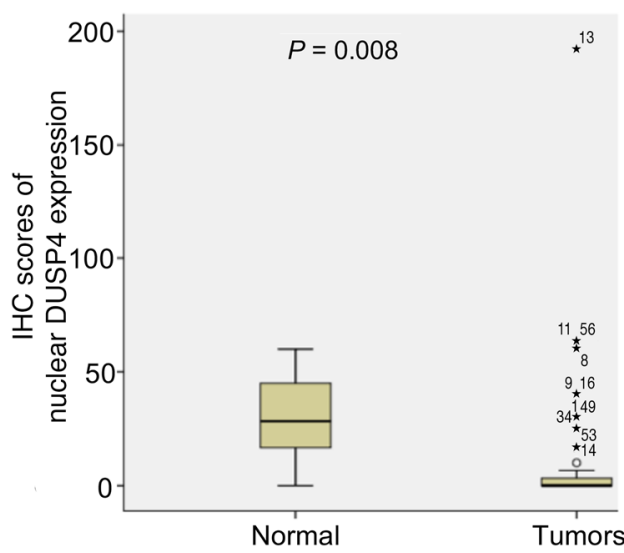

D

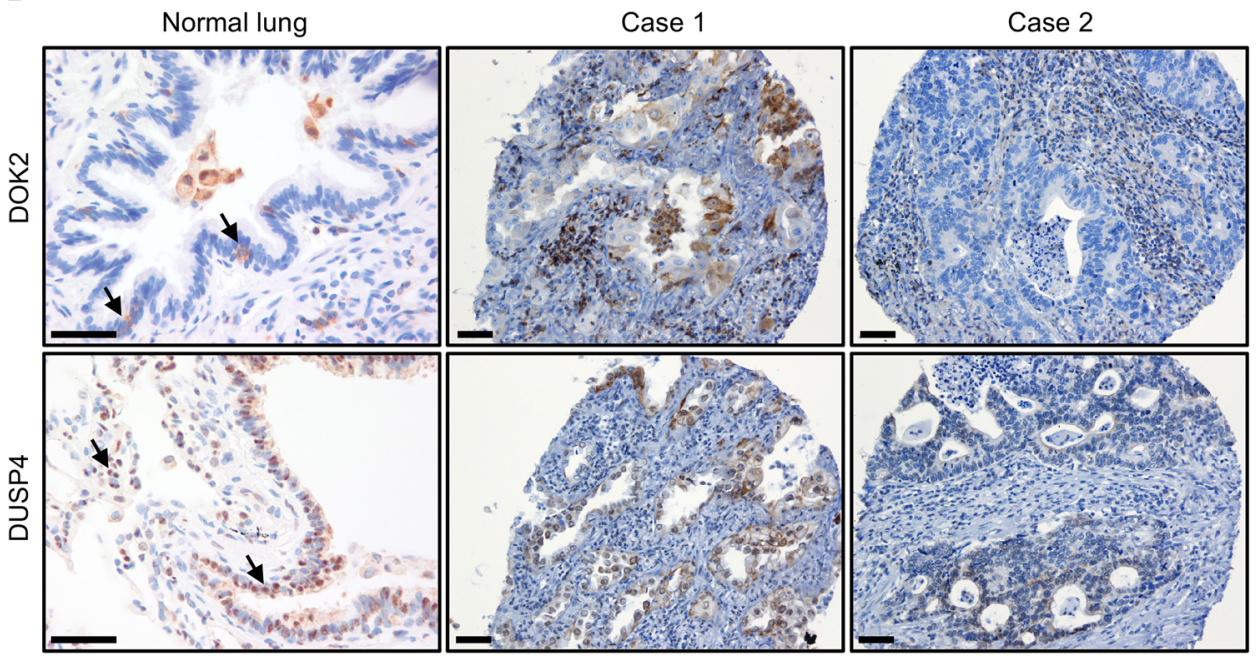

E

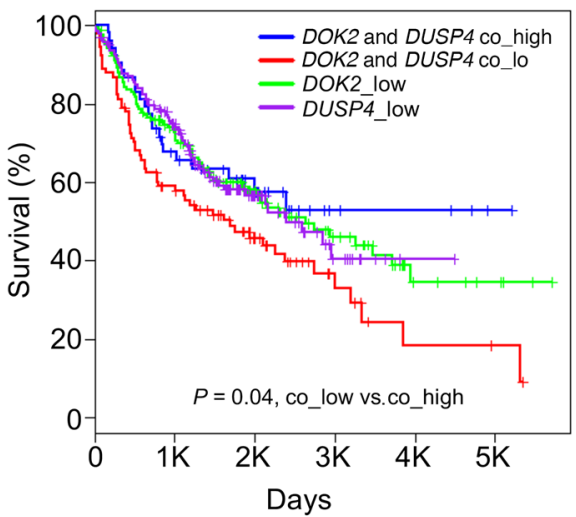

$\mathbf{F}$

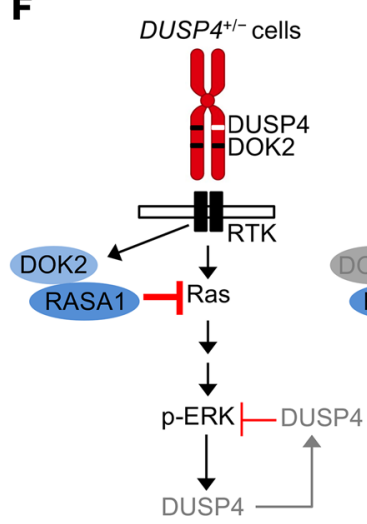

DOK2 $2^{+/-}$DUSP4 $4^{+/-}$cells

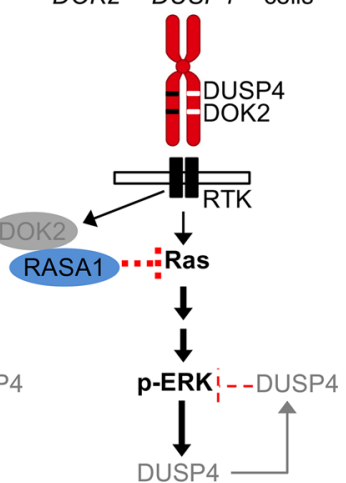

Figure 4. Compound loss of DOK2 and DUSP4 expression and its clinical implication in human lung adenocarcinomas. (A and $\mathbf{B}$ ) Percentage of tumors with deletion of DOK2 or DUSP4 (A) and co-deletion of DOK2 and DUSP4 (B) in the TCCA dataset. (C) DUSP4 protein expression in a TMA of normal lung tissue or lung cancer samples. $P$ values were determined by 2 -tailed $t$ tests. Data are shown as mean \pm SD. (D) Representative imagines of IHC staining for DOK2 and DUSP4 protein from normal lung tissue or 2 pairs of tumor samples with moderate or low expression of DOK2 and DUSP4 protein. Arrows indicate positive staining of DOK2 or DUSP4 protein in lung epithelial cells. Scale bars: $50 \mu \mathrm{m}$. (E) Kaplan-Meier survival curve of lung adenocarcinoma patients stratified by $D O K 2$ and DUSP4 expression. $P$ values were determined by log-rank test. (F) Models of compound haploinsufficiency of DUSP4 and DOK2 promoting lung tumorigenesis. DSUP4heterozygous cells: The activation of RTK signaling is tightly controlled by a negative feedback loop involving the recruitment of DOK2 and RASA1. The remaining WT DUSP4 allele is sufficient to suppress the low level of ERK activity transduced by RTK signaling. DUSP4 and DOK2 compound-heterozygous cells: Loss of DOK2 results in decreased recruitment of RASA1, allowing enhanced RTK and downstream ERK activation. The remaining WT DUSP4 allele is insufficient to suppress the high level of ERK activity and leads to increased lung tumorigenesis. White rectangles indicate genomic deletions; gray text indicates low expression of proteins of interest. data suggest that compound haploinsufficiency might serve as a biomarker to predict responsiveness to anticancer therapies.

Compound loss of DOK2 and DUSP4 predicts poor survival in human lung adenocarcinoma. To assess the relevance of these findings to human lung adenocarcinoma, we analyzed copy number of DOK2 and DUSP 4 in 513 human lung adenocarcinoma samples from The Cancer Genome Atlas (TCGA). Copy number loss of DOK 2 and DUSP4 was observed in $52 \%$ and $50 \%$ of cases, respectively, the majority of which were single copy losses (Figure $4 \mathrm{~A}$ and Supplemental Figure 2A). Co-loss of DOK2 and DUSP4 occurred in $49 \%$ of cases, and compound-heterozygous loss of DOK2 and DUSP 4 accounted for $90 \%$ of these co-deletion cases (Figure 4B). Copy number loss of DOK2 or DUSP4 was correlated with low expression of their respective mRNAs (Supplemental Figure 2B), suggesting that copy number alterations deregulate the level of DOK2 and DUSP4 in lung adenocarcinoma.

To evaluate DOK2 and DUSP4 protein expression in human lung tumors, we performed tissue microarray (TMA) analysis in 57 lung tumors (Supplemental Table 2). DOK2 expression was at a low or undetectable level in the majority of tumor samples (Sup- 
plemental Table 2). Likewise, DUSP4 protein expression was significantly lower in tumor tissue compared with the noninvolved normal lung (Figure 4C and Supplemental Table 2). Tumors with low expression of both DOK2 and DUSP4 protein accounted for $63 \%$ (34 of 54) of tumor samples examined (Supplemental Table 2 and Figure 4D).

To determine whether compound loss of DOK2 and DUSP4 impacts clinical outcome, we analyzed whether downregulation of DOK2 and DUSP4 is associated with survival outcome in human lung adenocarcinoma. We analyzed gene expression data from 764 lung cancer patients with available survival data (18-22). We found that although low expression of either DOK2 or DUSP4 alone had no association with survival outcome (Figure 4E), concomitant low expression of DOK2 and DUSP4 was significantly associated with poor survival (Figure 4E). Thus, compound loss of DOK2 and DUSP4 may be a predictor of aggressive disease in human lung cancer. Consistent with these findings, the association between copy number loss of DOK2 or DUSP4 alone and patient survival only approached significance (Supplemental Figure 2C). Taken together, these data indicate that concomitant downregulation of DOK2 and DUSP4 promotes lung tumorigenesis.

\section{Discussion}

Our study provides direct evidence for a model in which compound haploinsufficiency contributes to tumor progression as a result of heterozygous chromosomal deletions (Figure 4F). We found that in mice, loss of Dusp4 alone is insufficient to initiate cancer in the lung, which could be due to the redundancy in the Dusp protein family. However, heterozygous loss of Dusp4 did accelerate the onset and incidence of lung adenocarcinoma initiated by Dok2 heterozygosity. This work highlights the utility of mouse models and functional assays in disentangling the genetic effects of SCNAs. Future efforts should be therefore directed toward defining the combinatorial genetic interactions in recurrent cancerassociated somatic alterations, facilitated by TALEN or CRISPR/ Cas9 technology that can now inactivate multiple genes simultaneously in heterozygosity $(8,23)$.

This study lends further support to the notion that recurrent broad-scale deletions in human cancer, including $3 p, 5 q, 8 p$ 9p, and $17 p$ (1), play a critical role in tumorigenesis. Our work adds to a body of evidence supporting our hypothesis that loss of one allele, or reduced expression, of a critical TSG is functionally important either intrinsically or in combination with mutation of other genes $(4,5,24)$.

Our results demonstrate that compound haploinsufficiency can lead to activation of the convergent pathway and confer enhanced sensitivity to drugs inhibiting the aberrantly activated pathway. While much work is needed to translate the therapeutic implications of compound haploinsufficiency to the clinic, the high frequency of these genomic events in human cancer warrants further investigation. Finally, asitis a recentlyidentified lung TSG, the geneticinteractions of DOK2 with its various effectors, including RASA1/RASGAP (Figure $4 \mathrm{~F}$ ), should be further explored. For instance, loss or mutation of RASA1 is observed in $2 \%$ of lung adenocarcinoma, which would be predicted to disrupt DOK2's ability to suppress MAPK signaling, hence defining a novel subtype of lung cancer that is highly sensitive to MEK inhibition (25). Therefore, understanding the relationship between $8 \mathrm{p}$ genes and their effectors might also help facilitate patient stratification and the development of more effective cancer treatments.

\section{Methods}

Mice and histopathological analyses. All mouse strains have been previously described $(16,26)$. Dusp4-KO mice originally generated in a mixed C57BL/6 and 129/Sv genetic background were backcrossed to a $129 /$ Sv background by successive mating of Dusp4-heterozygous male mice to $129 / \mathrm{Sv}$ female mice and then maintained on a $129 / \mathrm{Sv}$ background as Dok2-KO mice. Total body necropsy and histopathological analyses were performed on cohorts of male and female mice at 9,12, and 18 months of age. Mouse tissues were fixed in $4 \%$ paraformaldehyde, embedded in paraffin, and sectioned and stained with $\mathrm{H} \& \mathrm{E}$ for pathological analyses.

Cell lines, Western blotting, and immunohistochemistry. All cell lines were obtained from ATCC and checked for mycoplasma by use of a MycoAlert Mycoplasma Detection Kit (Lonza). Cells and tissues were lysed with RIPA buffer (50 mM Tris [pH8], $150 \mathrm{mM} \mathrm{NaCl}, 0.1 \%$ SDS, $0.5 \%$ sodium deoxycholate, $1 \%$ NP40, 1 mM EDTA, and protease and phosphatase inhibitor cocktail [Roche]). For Western blotting, the following antibodies were used. Anti-phospho-ERK (catalog 9101) and anti-ERK (catalog 9102) were from Cell Signaling Technology. Anti-DUSP4 (S-18) and anti-HSP90 (H-114) were from Santa Cruz Biotechnology. Anti- $\beta$-actin (A3853) was from Sigma-Aldrich. Densitometry quantification was performed with NIH ImageJ. For immunohistochemistry, anti-phospho-ERK (20G11) was from Cell Signaling Technology. Anti-Ki-67 (SP6) was from Thermo Fisher Scientific. Anti-DUSP4 (10739-1-AP) was from Proteintech. Anti-DOK2 for both applications was provided by B. Clarkson (Memorial Sloan Kettering Cancer Center, Molecular Pharmacology and Chemistry Program, New York, New York, USA). See complete unedited blots in the supplemental material.

Generation of lung cancer cell lines stably overexpressing DOK2 or/and DUSP4, and cell growth analysis. Retrovirus was generated by transfection of pCL-Ampho and pBabe-puro-DOK2 or pBabe-hygro-DUSP4 into 293T cells. Forty-eight and 72 hours after transfection, culture supernatants were filtered, supplemented with Polybrene, and added to lung cancer cell lines. Forty-eight hours after the last infection, cells were selected for 2 days in media containing $2 \mu \mathrm{g} / \mathrm{ml}$ puromycin or $100 \mu \mathrm{g} / \mathrm{ml}$ hygromycin before use in experiments. For growth curve analysis, $1.5 \times 10^{4}$ cells per well were plated into 12 -well plates. At the indicated times, cells were washed with PBS, fixed in $10 \%$ formalin, and rinsed with distilled water. Cells were stained with $0.1 \%$ crystal violet, rinsed extensively, and dried. Cell-associated dye was extracted with $1.0 \mathrm{ml}$ of $10 \%$ acetic acid and then diluted, and the optical density (OD) at $595 \mathrm{~nm}$ was determined. Within an experiment, each point was determined in triplicate. The growth curve experiment was repeated 3 times.

TMA analysis. Sections of formalin-fixed, paraffin-embedded lung surgical specimens from 57 patients with lung cancer were stained with $\mathrm{H} \& \mathrm{E}$ and reviewed to identify viable, morphologically representative areas of normal lung and tumor areas from which needle core samples were taken. From each specimen, triplicate tissue cores with diameters of $1 \mathrm{~mm}$ were punched and arrayed onto a recipient paraffin block using a precision instrument (Beecher Instruments). Fivemicrometer sections of these TMA blocks were stained with H\&E for morphology assessment and further used for IHC analysis. 
Copy number, gene expression, and survival analysis. For the copy number variation analysis, we downloaded data from the TCGA database. The cutoff thresholds we used were -0.3 for deletions and 0.6 for amplifications; those lower than -0.8 were considered as homozygous deletions and higher than 0.8 as 2-copy amplifications. GISTIC (Genomic Identification of Significant Targets in Cancer) analysis was conducted to identify the copy number alterations of the chromosome $8 \mathrm{p}$ genomic region in TCGA lung adenocarcinoma samples. Deletion of many regions of chromosome $8 \mathrm{p}$ was detected across TCGA lung adenocarcinoma samples. The estimated FDR ( $q$ values) for chromosome 8 p deletion was drawn along the chromosome coordinates (27). A $t$ test was used to compare mRNA expression of DOK2 or DUSP4 against its copy number change among the homozygous, heterozygous, and noalteration groups. For the gene expression and survival analysis of human samples, we collected lung cancer microarray raw datasets from the NCBI GEO, including GSE31210, GSE37745, GSE19188, GSE29013, and GSE3141. All of these data were from the Affymetrix U133 plus 2.0 platform. We conducted gene expression data normalization together. Patient samples were ranked by the expression of the DOK2 gene from low to high. The first one-third of patients were considered as DOK2-low, the last one-third were considered as DOK2-high. A similar classification of patient samples was conducted for the DUSP4 gene. The "co-low" group ( $n=96$ samples) was derived from the overlap of DOK2-low ( $n=164$ samples) and DUSP4-low ( $n=164)$; the "co-high" group ( $n=65$ samples) from overlap of DOK2-high and DUSP4-high. Survival analysis was conducted with the CRAN R packages "survminer" and "survival." We performed pairwise comparisons in survival analysis among 4 groups, with corrections for multiple testing.

Statistics. For analysis of continuous data, datasets were compared using unpaired 2-tailed Student's $t$ tests. For analysis of categorical data (for example, cancer incidence), $2 \times 2$ contingency tables were constructed, and datasets were compared using Fisher's exact test. For analysis of cell growth data, datasets were compared using 2-way ANOVA with Bonferroni's post hoc tests. $P$ values less than 0.05 were considered statistically significant. All statistical tests were executed using GraphPad Prism software or the statistical software R (version 3.1.2).
Study approval. Mouse studies were approved by the BIDMC IACUC under protocol 082-2014. The TMA used in this study was constructed at the Icahn School of Medicine at Mount Sinai following an IRB-approved protocol. Written informed consent was obtained from patients for use of pathologic tissue.

\section{Author contributions}

MC, MSD, CN, JF, MCM, and THT performed the experiments. $\mathrm{MC}, \mathrm{AHB}$, and PPP conceived and designed the experiments. $\mathrm{RP}, \mathrm{CCC}$, and PPP supervised the study. JZ performed all bioinformatic analyses. RTB conducted pathology analyses of mouse tissues. MC, JZ, MCM, and PPP analyzed the data. MC, JZ, AHB, and PPP wrote the manuscript. All authors critically discussed the results and the manuscript.

\section{Acknowledgments}

We would like to thank all the members of the Pandolfi laboratory for critical comments, and Lauren Southwood and Elizabeth Stack for editing the manuscript. We thank the BIDMC Histology Core and the Biorepository and Pathology Core of the Icahn School of Medicine at Mount Sinai for histological and immunohistochemical analyses. This work was supported by NIH grant R01CA142787 to PPP. MC was supported in part by the Lung Cancer Research Foundation. AHB was supported by a postdoctoral fellowship from the American Cancer Society and an NIH/NCI Pathway to Independence award.

Address correspondence to: Pier Paolo Pandolfi, Beth Israel Deaconess Medical Center, CLS Building, Room 401, 330 Brookline Avenue, Boston, Massachusetts 02215, USA. Phone: 617.735.2121; Email: ppandolf@bidmc.harvard.edu.

MC's present address is: Department of Pathology, Duke University School of Medicine, and Duke Cancer Institute, Duke University, Durham, North Carolina, USA.

AHB's present address is: Fred Hutchinson Cancer Research Center, Seattle, Washington, USA.
1. Beroukhim R, et al. The landscape of somatic copy-number alteration across human cancers. Nature. 2010;463(7283):899-905.

2. Knudson AG. Two genetic hits (more or less) to cancer. Nature Rev Cancer. 2001;1(2):157-162.

3. Boultwood J, et al. Gene expression profiling of CD34+ cells in patients with the $5 \mathrm{q}$ - syndrome. BrJ Haematol. 2007;139(4):578-589.

4. Berger AH, Knudson AG, Pandolfi PP. A continuum model for tumour suppression. Nature. 2011;476(7359):163-169.

5. Berger AH, Pandolfi PP. Haplo-insufficiency: a driving force in cancer. J Pathol. 2011;223(2):137-146.

6. Solimini NL, et al. Recurrent hemizygous deletions in cancers may optimize proliferative potential. Science. 2012;337(6090):104-109.

7. Liu Y, et al. Deletions linked to TP53 loss drive cancer through p53-independent mechanisms. Nature. 2016;531(7595):471-475.

8. Cai Y, et al. Loss of chromosome 8p governs tumor progression and drug response by altering lipid metabolism. Cancer Cell. 2016;29(5):751-766.

9. Xue W, et al. A cluster of cooperating tumor-suppressor gene candidates in chromosomal deletions. Proc Natl Acad Sci U S A. 2012;109(21):8212-8217.

10. Birnbaum D, Adélaïde J, Popovici C, Charafe-Jauffret E, Mozziconacci MJ, Chaffanet M. Chromosome arm 8p and cancer: a fragile hypothesis. Lancet Oncol. 2003;4(10):639-642.

11. Chitale D, et al. An integrated genomic analysis of lung cancer reveals loss of DUSP4 in EGFRmutant tumors. Oncogene. 2009;28(31):2773-2783.

12. Martinez-Climent JA, et al. Loss of a novel tumor suppressor gene locus at chromosome $8 \mathrm{p}$ is associated with leukemic mantle cell lymphoma. Blood. 2001;98(12):3479-3482.

13. Wistuba II, et al. Allelic losses at chromosome 8p21-23 are early and frequent events in the pathogenesis of lung cancer. Cancer Res. 1999;59(8):1973-1979.

14. Berger AH, et al. Identification of DOK genes as lung tumor suppressors. Nat Genet. 2010;42(3):216-223.

15. Berger AH, et al. DOK2 inhibits EGFR-mutated lung adenocarcinoma. PLOS ONE. 2013;8(11):e79526.

16. Al-Mutairi MS, et al. MAP kinase phosphatase-2 plays a critical role in response to infection by Leishmania mexicana. PLoS Pathog. 2010;6(11):e1001192.

17. Barretina J, et al. The Cancer Cell Line Encyclopedia enables predictive modelling of anticancer drug sensitivity. Nature. 2012;483(7391):603-607.

18. Botling J, et al. Biomarker discovery in non-small cell lung cancer: integrating gene expression profiling, meta-analysis, and tissue microarray validation. Clin Cancer Res. 2013;19(1):194-204.

19. Okayama $H$, et al. Identification of genes upregulated in ALK-positive and EGFR/KRAS/ ALK-negative lung adenocarcinomas. Cancer Res. 2012;72(1):100-111.

20. Xie Y, et al. Robust gene expression signature 
from formalin-fixed paraffin-embedded samples predicts prognosis of non-small-cell lung cancer patients. Clin Cancer Res. 2011;17(17):5705-5714.

21. Hou J, et al. Gene expression-based classification of non-small cell lung carcinomas and survival prediction. PLOS ONE. 2010;5(4):e10312.

22. Bild AH, et al. Oncogenic pathway signatures in human cancers as a guide to targeted therapies. Nature. 2006;439(7074):353-357.
23. Hsu PD, Lander ES, Zhang F. Development and applications of CRISPR-Cas9 for genome engineering. Cell. 2014;157(6):1262-1278.

24. Alimonti A, et al. Subtle variations in Pten dose determine cancer susceptibility. Nat Genet. 2010;42(5):454-458.

25. Hayashi T, Desmeules P, Smith RS, Drilon A, Somwar R, Ladanyi M. RASA1 and NF1 are preferentially co-mutated and define a distinct genetic subset of smoking-associated non-small cell lung carcinomas sensitive to MEK inhibition. Clin Cancer Res. 2018;24(6):1436-1447.

26. Niki M, et al. Role of Dok-1 and Dok-2 in leukemia suppression. JExp Med. 2004;200(12):1689-1695.

27. Beroukhim R, et al. Assessing the significance of chromosomal aberrations in cancer: methodology and application to glioma. Proc Natl Acad Sci US A. 2007;104(50):20007-20012. 